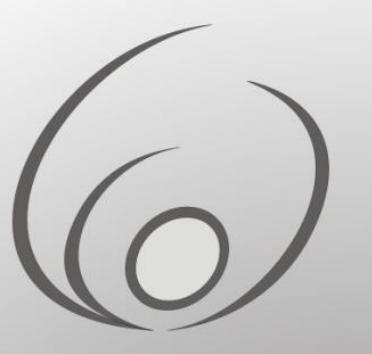

Fórum de

Pró-Reitores

de Extensão

das Instituições

Públicas de

Educação Superior

Brasileiras

\title{
A Liga de Neurociências: a complementação acadêmica nos estudos neurológicos com base em ações de ensino, pesquisa e extensão
}

\author{
Carlos Henrique F. Camargo ${ }^{1}$ \\ João Remí de Freitas Júnior ${ }^{2}$ \\ Karine Cim Assenço ${ }^{3}$ \\ Eduardo Antunes Martins ${ }^{4}$ \\ Marcelo Rezende Young Blood ${ }^{5}$
}

Resumo: A Liga Acadêmica de Neurociências é um projeto extensionista composto por um grupo de estudantes, coordenado por professores da UEPG - Universidade Estadual de Ponta Grossa, dedicados a se aprofundarem nas ciências neurológicas, ampliarem o conhecimento nas principais doenças da área das neurociências e atenderem as demandas da população sobre o tema. Os membros são designados por meio de um processo seletivo que ocorre anualmente. A liga abrange as três modalidades clássicas de aprendizado: Ensino, Pesquisa e Extensão. Na área de Ensino, há reuniões semanais sobre um assunto preestabelecido nas áreas de neurologia clínica, psiquiatria e neurocirurgia. Na Pesquisa, os alunos que apresentam interesse realizam projeto em iniciação científica sob a coordenação dos professores responsáveis pela liga. Por fim, na Extensão, os acadêmicos acompanham atendimentos nos ambulatórios de Neurologia do HURCG - Hospital Universitário Regional dos Campos Gerais - e praticam atividades direcionadas para a população, como palestras sobre AVC (Acidente Vascular Cerebral). A cada ano, novas ideias têm sido implantadas para enriquecer o conhecimento dos acadêmicos.

Palavras-chave: Instituições Acadêmicas, Neurociências, Educação Superior, Educação de Graduação em Medicina, Relações Comunidade-Instituição.

\footnotetext{
${ }^{1}$ Professor Adjunto do Departamento de Medicina da UEPG - Universidade Estadual de Ponta Grossa, Coordenador da Liga Acadêmica de Neurociências (LAN-UEPG) e autor para correspondência - chcamargo@uol.com.br

${ }^{2}$ Acadêmico do Curso de Medicina da UEPG - junior_de_freitas@hotmail.com

${ }^{3}$ Acadêmico do Curso de Medicina da UEPG - karine.kca@gmail.com

${ }^{4}$ Acadêmico do Curso de Medicina da UEPG - duduu_am@hotmail.com

${ }^{5}$ Professor Colaborador do Departamento de Medicina da UEPG e Supervisor da LAN-UEPG - myblood@gmail.com
} 


\title{
The Neuroscience League: the academic complementation at neurological studies based on
} actions of education, research and extension

\begin{abstract}
The Neuroscience Academic League is a university extension project composed of students supervised by professors from UEPG (Universidade Estadual de Ponta Grossa, Paraná, Brazil). This group is dedicated to delving into neurosciences, expanding their knowledge in the fields of major neuroscience-related diseases, as well as meeting the demands of the population on the subject. The members of the project are appointed through a selection process which takes place annually. The league covers the three classical learning methods: Teaching, Research and Extension. In the teaching, there are weekly meetings on predetermined subject fields of clinical neurology, psychiatry and neurosurgery. In the research, students who show interest can take part in undergraduate projects under the supervision of the professors in charge of the league. And finally, in the university extension the students can provide assistance in the Neurology ambulatories at HURCG (Hospital Universitário Regional dos Campos Gerais) and develop other activities such as lectures to the general public on the topic of cerebrovascular accidents (CVA). Every year, new ideas have been implemented to enrich the knowledge of the participants involved.
\end{abstract}

Keywords: Academic Institutions, Neurosciences, Higher Education, Undergraduate Medical Education, CommunityInstitutional Relations.

\section{La Liga de Neurociencia: los estudios de complementación académica en neurologia}

\section{basados en acciones de docencia, investigación y extensión}

Resumen: La Liga Académica de Neurociencias es un proyecto de extensión que comprende un grupo de alumnos, coordinados por profesores de la UEPG - Universidade Estadual de Ponta Grossa, Paraná, Brasil, dedicado a profundizar en las neurociencias, ampliar los conocimientos en el área de las enfermedades principales relacionadas con las neurociencias y satisfacer las demandas de la población sobre el tema. Los miembros son nombrados a través de un proceso de selección que se lleva a cabo anualmente. La liga cubre los tres métodos clásicos de aprendizaje: Enseñanza, Investigación y Extensión. En la Enseñanza, hay reuniones semanales en áreas predeterminadas de clínica de neurología, psiquiatría y neurocirugía. En la Investigación, los estudiantes que tienen interés realizan proyectos de investigación, bajo la supervisión de los profesores de la liga. Por último, en la extensión, académicos acompañan asistencias en las consultas externas de Neurología en HURCG - Hospital de Campos Gerais de la Universidade Regional - y participan en actividades dirigidas a la población como conferencias sobre accidentes cerebrovasculares. Cada año, nuevas ideas se han aplicado para enriquecer el conocimiento de los académicos.

Palabras-clave: Instituciones Académicas, Neurociencias, Educación Superior, Educación Médica de Pregrado, Relaciones comunitario-institucionales.

\section{Introdução}

As Neurociências compõem uma das bases disciplinares da graduação em Medicina, correspondendo a uma das mais complexas áreas do conhecimento em saúde, que demanda não apenas dedicação e entusiasmo por parte do graduando, mas principalmente de raciocínio lógico sequencial, no intuito de, efetivamente, obter a construção do saber. Frente ao modelo de assistência sanitária implantado no país, onde ainda persistem tantos pontos insatisfatórios, e a grande influência das instituições públicas de ensino sobre as comunidades que a permeiam, as ligas acadêmicas servem como importante ferramenta de extensão universitária na área da saúde.

Uma liga acadêmica de medicina é uma associação civil científica livre, de duração indeterminada, sem fins lucrativos, com sede e foro na cidade da instituição de ensino que a abriga, que visa complementar a formação acadêmica em uma área específica do campo médico, por meio de atividades que atendam aos princípios do tripé universitário de ensino, pesquisa e extensão (ASSOCIAÇÃO BRASILEIRA DE LIGAS ACADÊMICAS DE MEDICINA, 2010). Corresponde também a uma entidade estudantil destinada ao aprofundamento em determinada área do conhecimento 
para sanar demandas sociais (HAMAMOTO FILHO et al., 2011). No curso de medicina, as ligas têm particular importância, preenchem eventuais carências da graduação e contribuem para a melhoria dos serviços de saúde prestados na comunidade, além de promoverem a inserção dos acadêmicos e da instituição na sociedade (PERES et al., 2007).

Segundo Tavares et al. (2004), as atividades extensionistas são extremamente comuns no Brasil e constituem uma via importante no treinamento da maioria dos estudantes de medicina, contribuindo para a complementação da sua formação. Além disso, a extensão pode trazer uma rica experiência acumulada: o deslocamento do eixo pedagógico clássico professor aluno para o eixo aluno - comunidade, com um novo conceito de sala de aula; com a atuação do professor como co-participante, orientador, tutor, pedagogo; e com a ampliação do conceito de educador - por atuar em redes sociais - para o de uma rede de educadores (CORRÊA, 2003).

As neurociências clínicas compreendem as especialidades médicas neurologia, psiquiatria e neurocirurgia. Os conceitos básicos dessas áreas devem ser propriedade de todos os médicos, independentemente da especialidade que atuem, pois constituem doenças comuns no cotidiano médico. A neurologia é o ramo da medicina que trata dos distúrbios do sistema nervoso, atuando no diagnóstico e no tratamento das doenças que envolvem o sistema nervoso central e periférico, dando a conduta e seguimento clínico para uma gama de afecções como cefaleias (dores de cabeça), doenças neurodegenerativas (doença de Parkinson, doença de Alzheimer), distúrbios do sono, epilepsia, entre outras. A neurocirurgia atua nas doenças do sistema nervoso que possam necessitar de uma abordagem cirúrgica para o seu manejo, dentre as quais tumores do sistema nervoso, doenças vasculares, traumas crânio-encefálicos e lesões raqui-medulares. A psiquiatria tem sua atuação na prevenção, diagnóstico e tratamento dos distúrbios mentais, sejam de cunho orgânico ou funcional (BACHESCHI; GUERREIRO, 2004, HOPEN; VANZ, 2014).

Nas últimas décadas, a investigação dedicada às doenças neurológicas criou uma nova realidade. Os estudos identificaram agentes etiológicos, fatores de risco e mecanismos fisiopatológicos. Foram desvendadas bases genéticas e o seu papel em diversas doenças neurológicas. Esses avanços possibilitaram o desenvolvimento de novas terapêuticas, mais específicas e eficazes. Definiram-se critérios de diagnóstico padronizados que revelaram diferenças significativas na distribuição dessas doenças, principalmente entre nações desenvolvidas e em vias de desenvolvimento. Observouse um crescimento das doenças neurodegenerativas e cerebrovasculares com o envelhecimento da população e, com isso, o aumento de sua morbidade e mortalidade (ATALAIA-SILVA et al., 2008, EU JOINT PROGRAMME, 2014).

As características peculiares das doenças neurológicas colocam diversas dificuldades ao seu estudo e manejo, nomeadamente: muitas doenças neurológicas são raras; os sintomas e sinais paroxísticos de algumas patologias são comuns, tornando difícil determinar o início e perfil temporal; o período de latência até a sua apresentação clínica e ao diagnóstico pode ser longo; os critérios de diagnóstico variam entre estudos e com o tempo, dificultando a comparação de resultados; pode ser necessária a avaliação pos-mortem para o diagnóstico definitivo. $\mathrm{O}$ diagnóstico em vida exige, muitas vezes, o exame por um neurologista. Em muitas doenças não existem testes de diagnóstico confirmatórios e a análise de tecidos é difícil em vida. A fundamentação para o diagnóstico da maioria apoia-se na história clínica e no conhecimento de sua fisiopatologia (FERREIRA, 2011).

Por estes motivos, impõe-se a necessidade da discussão das doenças neurológicas prevalentes, a fim de se ampliar o conhecimento sobre as neurociências, buscando desenvolver a capacidade de maior acurácia diagnóstica e resolutividade terapêutica na formação dos futuros médicos.

Nesse sentido, a Liga Acadêmica de Neurociências (LAN) foi criada em 2011, com o objetivo de suprir lacunas da graduação geral, aproximar a instituição de ensino e os participantes da comunidade externa, levando educação em saúde à comunidade acadêmica e aos docentes, membros da UEPG, bem como à sociedade de modo geral, no que concerne à compreensão das principais doenças neurológicas, contribuindo para a efetividade dos serviços de saúde prestados na região, direta ou indiretamente.

Dentre os objetivos específicos da Liga, estão: (1) criar habilidades que consistem no conhecimento das manifestações clínicas das mais diversas síndromes neurológicas, na correta e adequada realização do exame físico neurológico e na capacidade de discernir sobre a conduta a ser tomada diante de cada caso clínico; (2) atender, de forma integrada e capacitada, os pacientes que forem de sua competência, visando o tratamento de seus agravos de saúde e o desenvolvimento de conhecimentos de promoção da saúde; (3) promover o encontro dos acadêmicos com os pacientes portadores de doenças neurológicas, visando uma melhor qualidade da relação médico-paciente, fundada nos princípios da ética e da humanização; (4) proporcionar aos alunos o amadurecimento acadêmico, com a elaboração e publicação de livros e artigos científicos; (5) trabalhar o relacionamento interpessoal entre os acadêmicos e outros profissionais da área da saúde; (6) buscar a compreensão dos temas abordados nas discussões clínicas.

Nas atividades extensionistas, o objetivo maior é aproximar a comunidade dos acadêmicos, fazendo com que os mesmos sejam capazes de sintetizar, simplificar e transmitir os seus conhecimentos a ela, levando para sua vida profissional uma experiência de boa relação com seu paciente. Além disso, objetiva-se proporcionar ao paciente um ambiente mais favorável ao tratamento de sua doença, criando-se ambientes férteis de discussão clínica entre acadêmicos e professores, a fim de buscar a melhor abordagem para a resolução de seus problemas. 


\section{Metodologia}

\section{Criação da Liga de Neurociências}

A Liga de Neurociências é um projeto de extensão do Departamento de Medicina, coordenado pelo Professor Doutor Carlos Henrique Ferreira Camargo, iniciado em março de 2011, e está registrada na Pró-Reitoria de Extensão da UEPG.

\section{Seleção dos acadêmicos para a Liga}

Os Acadêmicos interessados em participar da Liga são submetidos a um processo seletivo. Nos dois primeiros anos, os acadêmicos interessados realizaram uma prova de admissão e análise de curriculum vitae. No terceiro ano, os alunos foram selecionados enviando seu coeficiente de rendimento e a média adquirida na disciplina de neurociências. Os outros acadêmicos do Curso Medicina são convidados a participar das atividades da liga como ouvintes.

\section{Atividades da Liga - Reuniões para discussão de temas e casos clínicos}

As reuniões são abertas a toda a comunidade acadêmica da UEPG. Cada acadêmico participante da Liga de Neurociências da UEPG escolhe, inicialmente, um tema de sua preferência, no âmbito das áreas abrangidas por este projeto. De acordo com uma escala que intercala temas neurológicos, psiquiátricos e neurocirúrgicos, há uma apresentação semanal relativa a uma revisão da literatura do assunto escolhido, seguida pela apresentação de um caso clínico relevante ao tema estudado na semana anterior.

No período que precede a reunião pela qual se responsabiliza, o acadêmico é orientado pelos professores da liga a realizar a revisão literária, utilizando-se de artigos científicos publicados em revistas com maior impacto na comunidade científica. No caso da apresentação de casos clínicos, o professor que orienta o aluno pode sugerir um caso previamente conhecido por ele ou, ainda, fica a cargo do estudante revisar a literatura, encontrar um caso descrito por uma revista de grande impacto e elaborar uma apresentação.

Durante o primeiro semestre, as apresentações são elaboradas em português, e no segundo semestre devem ocorrer totalmente em inglês. No dia de sua apresentação, o aluno responsável ministra uma palestra de duração média de 60 minutos para os participantes, convidados especiais e comunidade acadêmica interessada. Após a apresentação, são tecidos comentários e críticas por médicos com entendimento no assunto e, posteriormente, há um espaço para discussão, de tempo variável.

Esta discussão abrange o tema da aula ministrada e complementações que os alunos integrantes da liga e os professores julgarem necessárias, de modo a unificar e perpetuar o conhecimento. As informações adquiridas durante as discussões científicas são trabalhadas de maneira que sejam, posteriormente, refletidas e repassadas à população pelas atividades de educação em saúde da liga ou durante as atividades ambulatoriais. Esses conteúdos servem de base, também, para o atendimento nos ambulatórios e para a organização dos eventos específicos tanto para a comunidade quanto para a comunidade acadêmica.

\section{Atividades da Liga - Atividades no ambulatório de Neurologia}

Em relação às atividades ambulatoriais, os integrantes são divididos em duplas e organizados em escalas para atendimentos supervisionados de pacientes com problemas neurológicos, nos ambulatórios especializados do HURCHG - Hospital Universitário Regional dos Campos Gerais. O atendimento no ambulatório ocorre todas as quartas-feiras no período das $7 \mathrm{~h} 30$ às $9 \mathrm{~h} 30$. A cada quarta-feira, esses dois acadêmicos participantes da LAN da UEPG acompanham os médicos neurologistas Dr. Carlos Henrique Ferreira Camargo e Dr. Marcelo Rezende Young Blood nas consultas médicas cabíveis a este ambulatório.

Os acadêmicos, então, têm a oportunidade de desenvolver a anamnese, isto é, a conversa e formulação da história clínica do paciente, além de realizar o exame físico geral e específico neurológico para aprender as manifestações clínicas neurológicas mais frequentes em cada doença. Eles ainda têm a oportunidade de formular hipóteses diagnósticas e tratamentos juntamente com o seu professor que, por fim, definirá a conduta a ser realizada para os pacientes atendidos.

Além disso, os acadêmicos desenvolvem habilidades como a redação de receitas médicas, requisição de exames e redação do atendimento aos pacientes no prontuário médico, guiados pelo médico responsável. Os pacientes atendidos nesse Ambulatório de Neurologia recebem, então, diagnóstico e conduta, além de informações sobre sua doença e sobre a prevenção de outros agravos e promoção da saúde. Após a despedida de cada paciente, o médico docente responsável inicia, junto aos acadêmicos, uma rápida discussão sobre os principais pontos.

\section{Projetos de orientação para a comunidade}

Durante o período de um ano são realizados pelo menos dois projetos de informação sobre as neurociências para a comunidade, em escolas e espaços públicos. A liga busca espaços que possam abranger um maior número de pessoas, como terminais rodoviários e praças, com uma abordagem individual, entrega de folders e explanação sobre o AVC para a população que passa por esses locais. 
Já nas escolas da rede pública, a abordagem é feita em outro momento, por meio de palestras ministradas pelos membros da liga para pais e professores, a respeito do TDAH (Transtorno de Déficit de Atenção e Hiperatividade), e uma roda de conversa com os adolescentes sobre problemas do seu dia a dia, como sexualidade, vocação profissional e interesses nessa fase da vida. Para o público leigo, na maioria das vezes, a compreensão das doenças neurológicas encontra-se seguramente longe de alcance, sendo as doenças que afetam o sistema nervoso consideradas as mais obscuras e preocupantes. É interessante levantar possibilidades que favoreçam a compreensão das neurociências não apenas no âmbito acadêmico, mas também na sociedade de modo geral.

\section{Projetos de ensino e pesquisa}

Os projetos de extensão são base para projetos de ensino e de pesquisa. Há reuniões para estabelecer as atividades na área de pesquisa, como os temas e a divisão dos mesmos na elaboração de livros e artigos científicos. Durante os dois últimos anos, desenvolveu-se um livro anualmente, com a participação de todos os membros da LAN. Para aqueles alunos que têm um interesse especial em pesquisa, os coordenadores da LAN os incluem em projetos de iniciação científica na área.

\section{Resultados e Discussão}

No sentido de aprimorar o ensino e a necessidade de formar profissionais como sujeitos ativos, com uma visão ampliada da saúde, respondendo às necessidades da população, a Extensão Universitária vem ganhando cada vez mais espaço como processo educativo, cultural e científico, que articula o Ensino e a pesquisa de forma indissociável e viabiliza a relação transformadora entre a Universidade e a sociedade (NOGUEIRA, 2000).

Os resultados da liga refletem-se no maior domínio técnico dos acadêmicos acerca das neurociências e suas ramificações, e na aquisição de experiência e sensibilidade dos mesmos na abordagem a pacientes neurológicos e no contato com a comunidade. $\mathrm{O}$ aprimoramento dos serviços de saúde fornecidos também é observado, considerando-se a inserção de profissionais gabaritados, que compõem o corpo docente, no serviço público de neurologia. Na área de ensino, as aulas têm sido ministradas com êxito pelos acadêmicos, que se mostraram capazes de ler e interpretar as informações contidas nos artigos científicos em língua estrangeira e transformá-las em aulas de forma coerente. As discussões se seguem de maneira bastante produtiva, com conteúdo claramente compreendido por ambos, apresentador e ouvintes.

Nas apresentações realizadas em inglês, no decorrer das semanas, foi possível evidenciar o impacto positivo desta medida no que tange a ansiedade esperada para a apresentação em uma língua diferente, assim como a melhora de compreensão e habituação aos temas por parte dos ouvintes. Observa-se, assim, uma forma de incentivo ao aprendizado instrumental do inglês em medicina, com o desenvolvimento de habilidades específicas necessárias ao aluno e também ao profissional médico.

Além da capacidade de ler e interpretar o conteúdo de artigos científicos, o médico também deve ser capaz de comunicar essas informações em uma língua estrangeira. A importância dessas habilidades para o profissional da saúde é grande, e se baseia no princípio de que os periódicos médicos com os maiores fatores de impacto (New England Journal of Medicine, The Lancet, Journal of the American Medical Association, Annals of Internal Medicine) são publicados somente em inglês, e tais informações são extremamente valiosas, tanto para o estudante em formação, durante a elaboração de pesquisas científicas e no próprio estudo, assim como para a educação continuada do médico já graduado, que precisa se abastecer de conhecimentos recentes.

Além disso, a necessidade da comunicação falada em inglês se dá pelo fato de que o estudante, com esta habilidade, é capaz de participar de congressos internacionais, como ouvinte, e também como apresentador de trabalhos científicos. O médico graduado pode, assim, usufruir com mais facilidade de congressos internacionais para manter-se atualizado, sendo também capaz de expor seus conhecimentos e indagações em língua estrangeira.

O ganho com o ambulatório da Liga tem sido visível em função de um maior desenvolvimento de conhecimentos científicos acerca das doenças neurológicas pelos acadêmicos participantes. Dessa forma, a cada dia tornam-se mais aptos a prestar assistência a pacientes que venham a apresentar alguma das doenças presenciadas no ambulatório. Além disso, os pacientes têm sido bem assistidos pelos acadêmicos, o que evidencia uma melhoria na relação médico-paciente e na relação acadêmico-paciente.

$\mathrm{O}$ atendimento ambulatorial também traz benefícios à comunidade, apresentando-se de duas maneiras distintas. Primeiramente, o benefício direto é concedido a partir do próprio atendimento médico que, ao ser realizado conjuntamente com os acadêmicos, se torna mais global, tendo em vista que os estudantes estão na fase de descoberta de conhecimentos e a discussão dos casos gera maiores possibilidades diagnósticas e de tratamento. A proximidade com o meio científico concede aos atendimentos uma maior capacidade resolutiva, visto que meios diagnósticos disponíveis na universidade podem ser acessados de maneira mais rápida e contribuir para o manejo clínico.

De maneira indireta, a comunidade será favorecida através da formação mais humana e otimizada de futuros profissionais médicos, pois o contato com o/a paciente desde as primeiras séries propicia um melhor entendimento da relação médico-paciente e a maneira com que os fatores sociais podem interferir no processo saúde-doença. Além disso, a presença de ambulatórios acadêmicos se mostra como um berço fértil de discussão científica, que aventa diagnósticos diferenciais para o 
paciente e atualizações nas terapêuticas propostas, promovendo também um maior acesso aos ambulatórios de especialidades pertencentes ao HURCG, necessários ao atendimento integral do paciente.

Dessa maneira, a universidade atua devolvendo para a sociedade os recursos a ela destinados, na forma de benefícios e avanços nos sistemas de saúde, no atendimento mais humano da população e na promoção integrada da saúde. Além disso, o conhecimento das doenças neurológicas, seus sintomas e possíveis tratamentos, levado até a população por meio das atividades de educação em saúde da liga, pode ajudar na prevenção das doenças e no atendimento mais precoce. Essa visão mais acadêmica e o estímulo de toda a equipe de saúde, com o entusiasmo adquirido no processo, acabaram melhorando o atendimento tanto qualitativa quanto quantitativamente (TORRES, 2008).

Outro resultado relevante é a maior realização de projetos de pesquisa, constituindo novas fontes de conhecimento pertinentes.

Com vistas à edição do livro Neurologia e Cinema, foi realizada uma síntese a partir da discussão de cada filme, levando-se em conta seus aspectos culturais e clínicos. No momento, a obra encontra-se me processo de revisão para posterior publicação.

A partir das discussões, os acadêmicos puderam aprofundar seus conhecimentos frente à doença neurológica em questão, buscando aspectos fisiopatológicos, sintomatológicos e de tratamento. Além disso, tiveram contato com grandes obras do cinema mundial, podendo analisar e aprofundar seus saberes neuro-científicos frente à atuação dos atores, buscando aspectos de interpretações que levassem ao diagnóstico em questão, bem como a maneira como a patologia foi abordada e seu desfecho na vida do personagem.

A segunda publicação está em processo de produção e se refere a um manual da semiologia e exame neurológico, voltado aos acadêmicos dos cursos de saúde, em virtude de que se trata de um tema bastante complexo e necessário para a formação do raciocínio clínico, além de amplamente cobrado em provas e concursos de residência médica. Espera-se, até o final de 2014, a publicação dessas duas obras voltadas à comunidade acadêmica e leiga, que, além de ser um incentivo à cultura, serve como ferramenta de promoção de saúde, gerando a difusão dos conhecimentos das neurociências e promovendo um melhor entendimento do público diante das doenças neurológicas. Isso também permite a discussão, a aceitação e o enfrentamento da sociedade frente às dificuldades que os pacientes portadores dessas desordens sofrem em seu dia a dia.

Além disso, é evidente o impacto direto sobre a sociedade, trazido pela aproximação com a instituição de ensino, culminando em um coletivo mais consciente e satisfeito com o apoio oferecido.

Durante os três anos de Liga de Neurociências foram realizadas semanas de conscientização quanto ao
Acidente Vascular Cerebral (AVC) e reuniões de aconselhamento quanto ao TDAH nas escolas. Em grupos separados, professores e acadêmicos puderam organizar aulas expositivas e oficinas de discussão com professores/pedagogos, pais ou alunos. Os educadores apresentaram problemas observados na maioria das escolas que dificilmente conseguem receber solução: numa classe grande, existe dificuldade de se dar uma atenção especial a somente um aluno com desatenção; é muito difícil conseguir respeito dos alunos e, com isso, a sua atenção; muitas vezes, os alunos já vêm com déficits de aprendizagem dos anos anteriores, e por causa disso sentem-se incapacitados, pois há dificuldade de aprender.

Os pais, como esperado, tinham pouca informação sobre o TDAH e, ainda, a visão distorcida - promovida pela mídia e pela dificuldade cultural - em aceitar a existência de doenças neurológicas e psiquiátricas em crianças. Porém, após o momento de exposição, puderam apresentar suas dúvidas e até reconhecer seus filhos em vários dos critérios de diagnóstico de TDAH, baseados no comportamento e no rendimento escolar.

Por sua vez, aos adolescentes foi oportunizado o questionamento sobre dúvidas comuns nessa idade. A maioria das perguntas foi sobre doenças sexualmente transmissíveis, câncer de mama, gravidez na adolescência, uso de anticoncepcionais orais, mas também surgiram perguntas em relação à escolha da vocação profissional e carreira estudantil. Com o passar do tempo, os adolescentes ficavam menos encabulados em fazer perguntas e a maioria revelou que sente vergonha de falar sobre sexualidade com pais e professores, ao passo que ficam mais à vontade com um grupo de fora, como o formado pelos acadêmicos de medicina.

A Liga vem em processo constante de aprimoramento, promovendo mais iniciativas comunitárias e acadêmicas e, consequentemente, trazendo a cada dia mais impacto e resultados positivos tanto para os membros da Liga quanto para a universidade e para a comunidade.

\section{Conclusões}

Resta evidente o eminente papel das Ligas Acadêmicas na formação de novos profissionais, bem como na aproximação entre os membros do meio acadêmico e da comunidade. A gama de atividades da LAN da UEPG garante sua influência benéfica na região, tanto pelo melhor atendimento prestado à saúde, quanto pela formação mais completa dos futuros médicos.

A maioria de seus resultados não é mensurável em curto prazo, mas a sua continuidade torna-los-á evidentes futuramente, em indicadores de saúde e profissionais competentes graduados na Universidade Estadual de Ponta Grossa. Dessa forma, munindo-se de ações de conscientização, projetos de pesquisa, reuniões acadêmicas e atividades ambulatoriais, assegura-se o impacto positivo, imediato e futuro, da Liga. 


\section{Referências}

ASSOCIAÇÃO BRASILEIRA DE LIGAS ACADÊMICAS DE MEDICINA. Diretrizes Nacionais de Ligas Acadêmicas de Medicina. 2010. Disponível em: < http://www.ablam.org.br/diretrizes_nacionais .html>. Acesso em: 20 fev. 2014.

ATALAIA-SILVA, K. C.; RIBEIRO, P. C. C.; LOURENÇO, R. A. Epidemiologia das demências. Revista Hospital Universitário Pedro Ernesto, v. 1, n.7, p. 46-71, 2008.

BACHESCHI, L. A.; GUERREIRO, C. A. M. Situação das neurociências no Brasil: neurociências clínicas. Ciência e Cultura, v. 56, n. 1, p. 25-26, 2004.

CORRÊAA, E. J. Extensão universitária, política institucional e inclusão social. Revista Brasileira de Extensão Universitária, v. 1, n.1, p. 12-15, 2003.

EU JOINT PROGRAMME. Neurodegenerative Disease Research. [s.n.]. Disponível em < http://www.

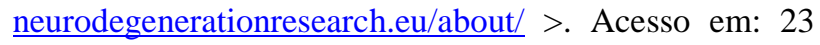
fev. 2014.

FERREIRA, L. R. O. Neuroepidemiologia no mundo: o particular em Portugal. Dissertação de Mestrado Integrado em Medicina, Universidade do Porto, Porto, Portugal, 2011.

HAMAMOTO FILHO, P. T.; VENDITTI, V. C.; OLIVEIRA, C. C.; VICENTINI, H. C.; SCHELLINI, S. A. Ligas Acadêmicas de Medicina: extensão das ciências médicas à sociedade. Revista Ciência em Extensão, v. 7, n. 1, p. 126-133, 2011.

HOPPEN, N. H. F; VANZ, S. A. S. Tendências da pesquisa brasileira em Neurociências. In: Encontro Brasileiro de Bibliometria e Cientometria (EBBC), 4., 2014, Recife, Pernambuco, Brasil, v. 4, POSTER-28, 2014, Disponível em < http://www.brapci.inf.br /_repositorio/2014/05/pdf_c714184cab_0014376.pdf>

Acesso em: 06 nov. 2014.

NOGUEIRA, M. D. P. Extensão Universitária: Diretrizes conceituais e Políticas. Belo Horizonte: PROEX / UFMG, 2000.

PERES, C. M.; ANDRADE, A. S.; GARCIA, S. B. Atividades extracurriculares: multiplicidade e diferenciação necessárias ao currículo. Revista Brasileira de Educação Médica, v. 31, n. 3, p. 203-211, 2007.

TAVARES, A. P.; CARDOSO, S. A. V.; DANTAS, N. G. T.; LOPES, G. C.; FONSECA JÚNIOR, C. A. F. O currículo paralelo dos estudantes de medicina e a extensão universitária. In: Congresso Brasileiro de Extensão Universitária, 2., 2004, Belo Horizonte, Minas Gerais, Brasil. Anais eletrônicos..., Belo Horizonte: UFMG, 2004. Disponível em: < https://www.ufmg.br/congrext/Educa /Educa116.pdf $>$. Acesso em: 23 fev. 2014.

TORRES, A. R.; OLIVEIRA, G. M. D.; YAMAMOTO, F. M.; LIMA, M. C. P. Academic Leagues and medical education: contributions and challenges. Interface Comunicação, Saúde, Educação, v.12, n. 27, p.713720, 2008.

Como citar este artigo:

CAMARGO, C. H. F.; FREITAS JÚNIOR, J. D.; ASSENÇO, K. C.; MARTINS, E. A.; YOUNG BLOOD, M. R. A Liga de Neurociências: a complementação acadêmica nos estudos neurológicos com base em ações de ensino, pesquisa e extensão. Revista Brasileira de Extensão Universitária, v. 5, n. 2, p. 47-53, 2014. Disponível em: https://periodicos.uffs.edu.br/index.php/RBEU/article/vie w/1135/pdf> 